\title{
KRIEGSLITERATUR AUS DER DDR UND DIE ZENSUR IN DER VOLKSREPUBLIK POLEN IN DEN ERSTEN NACHKRIEGSJAHREN
}

\begin{abstract}
GDR war literature and censorship in People's Republic of Poland in the early postwar years

From all of the German literature distributed in Poland during the first half of the nineteen fifties, that of the GDR was the most strongly represented, because like the People's Republic, it was part of the Eastern Bloc. A substantial part of this literature touched upon the themes of the Second World War. As some prominent Eastern German authors had taken part in the Spanish Civil War in 1936-1939, this subject also couldn't be ignored.

The introduction in 1949 of socialist realism as the most important criterion of art, and particulary strong political pressure, led to a great deal of confusion and insecurity, not only for Polish publishing houses, but also among the censors, whose task was to take decisions about what literature could be printed. Censors' opinions in this period often differed, not only in terms of detailed matter, but also in the final decisions about the eventual fate of the title submitted for evaluation.
\end{abstract}

KEY WORDS: Germane literature, war literature, censorship, socialist realism, GDR, Polish People's Republic

In der ersten Hälfte der 1950er Jahre, kaum zehn Jahre nach dem Ende des Zweiten Weltkriegs, konnten in der Volksrepublik Polen fast ausschließlich Werke von Autoren aus der Sowjetischen Besatzungszone bzw. aus der gerade erst gegründeten, ebenfalls zum Block der sozialistischer Staaten gehörenden DDR publiziert werden. Ein großer Teil der in dieser Zeit von den polnischen Verlagen zur Zensur eingereichten Prosawerke behandelte das Thema des vor kurzer Zeit beendeten Krieges. Da einige prominente ostdeutsche Autoren an dem Spanischen Bürgerkrieg in den Jahren 1936-1939 beteiligt waren, konnte auch dieses Thema nicht fehlen. Nicht alle Werke kommunistischer deutscher Schriftsteller konnten jedoch die Schranken der Zensur problemlos passieren. Die ideologische Strenge des 1949 ausgerufenen sozialistischen Realismus als führende künstlerische Methode und die 
politische Unsicherheit dieser Zeit führten nicht nur zu gewissen Strapazen der von den polnischen Kommunisten gegründeten und dem neuen System wohlwollend gegenüberstehenden Verlagen, sondern auch zur Verunsicherung der Zensoren. ${ }^{1}$

Unter den Prosawerken von DDR-Autoren, in denen der Krieg im Mittelpunkt steht, gibt es eine Reihe von Texten, die die einzelnen Zensoren zwar positiv beurteilten, so dass sie sich für die Erteilung der Druckgenehmigung ohne Eingriffe aussprachen, in ihre Gutachten aber mehr oder weniger kritische Bemerkungen einfügten. Bei der Betrachtung dieser Gutachten kommt die Vermutung auf, dass die jeweiligen kritischen, manchmal beiläufig gemachten Äußerungen die Funktion einer Absicherung der Zensoren gegenüber ihren Vorgesetzten hatten. Wie es scheint, wollten manche von ihnen gerade am Beginn der 1950er Jahre nicht den Eindruck erwecken, bei der Prüfung von Werken namhafter Autoren aus der DDR ideologische Fehler übersehen zu haben.

Der Roman Das siebte Kreuz von Anna Seghers, der vom Verlag Czytelnik herausgegeben werden sollte, ist ein gutes Beispiel für einen Text, dessen ideologische Mängel die Zensoren zwar aufmerksam registrierten, den sie aber dennoch zum Druck ohne irgendwelche Eingriffe freigaben. Das Gutachten vom 2. Juli 1951 kann geradezu als Paradebeispiel für das Vorgehen der Zensur in dieser Zeit im Hinblick auf literarische Texte gelten, in denen ein breites Spektrum der deutschen Gesellschaft dargestellt wird. Der Zensor lobt einerseits die unzweifelhaft hervortretenden positiven Seiten der Veröffentlichung des Romans:

Das Buch ist eine ausgesprochen positive Veröffentlichung, die mutig und klar die Herrschaft des Hitlerregimes und die Stimmungen in der deutschen Bevölkerung beleuchtet. (...) Die Übersetzung ist sehr gut, das Buch ist eine sehr interessante Veröffentlichung, die den Leser mit der Zeit des Hitlerregimes in Deutschland bekanntmacht. Es ist gegen den Faschismus gerichtet. (AAN GUKPPiW 375-31/27, Gutachten vom 2.7.1951)

Andererseits kommt er seinen Pflichten als Zensor nach und macht auf die vermeintlichen Schwächen des Werkes aufmerksam - Anna Seghers habe nämlich kein Bild von den Aktivitäten der deutschen Kommunistischen Partei, besonders ihrer Rolle bei der Aufklärung der Gesellschaft und bei der Mobilisierung der Deutschen zum Widerstand gegen den Nationalsozialismus, gegeben. (AAN GUKPPiW 375-31/27, Gutachten vom 2.7.1951) Es handelte sich dabei um ein Gutachten der Nachzensur, eventuelle Eingriffe in den Text waren nicht mehr möglich. Da eine Neuauflage des Romans vom Verlag in Aussicht genommen wurde und der Zensor sich in einem Punkt gegen den Roman äußerte, wurde ein weiterer Zensor mit der

\footnotetext{
${ }^{1}$ Der vorliegende Beitrag bezieht sich auf Quellenrecherchen, die im Archiwum Akt Nowych in Warschau durchgeführt wurden, und geht auf meine bereits veröffentlichte Studie zur Zensierung der deutschsprachigen Literatur in Polen und der polnischen Literatur in der DDR zurück. Die aus dieser Studie übernommenen Abschnitte wurden teilweise verändert und um neue Erkenntnisse und Feststellungen ergänzt. (Rajch, 2015).
} 
Kontrolle beauftragt. Sein Gutachten, in dem er auf die Bedenken seines Vorgängers nicht eingegangen ist, resümierte er kurz: „Neuauflage des bekannten Romans Das siebte Kreuz der deutschen Schriftstellerin Anna Seghers, den deutschen Arbeitern gewidmet, die gegen das Hitlerregime kämpften. Keine Bedenken gegen die Veröffentlichung des Romans.“ (AAN GUKPPiW 375-31/27, Gutachten vom 2.7.1951) Die Nachauflage des Romans konnte erfolgen.

Die von Willi Bredel stammenden Erinnerungen an den Spanischen Bürgerkrieg, die vom Państwowy Instytut Wydawniczy (PIW) in den Jahren 1949/1950 verlegt wurden, sind ein prägnantes Beispiel für Texte, die von der Zensur zwar als erwünscht aber auch eingriffsbedürftig betrachtet wurden. Zum ersten Mal wurde der Text Ende 1949 oder im Januar 1950 von einem Zensor geprüft, der keinen Grund zur Beanstandung fand und die Genehmigung zum Druck erteilte. Der Roman sei den polnischen Lesern zu empfehlen, weil der Autor in ,warmen und herzlichen Worten" den polnischen General Karol Świerczewski beschreibt. Da die Erinnerungen von Willi Bredel die Beteiligung der deutschen Antifaschisten auf der Seite der Republikaner zeigen, habe das Buch „eine große aktuell-erzieherische Bedeutung" (AAN GUKPPiW 152-31/120, Gutachten vom 2.1.1950). Der zweite Gutachter äußerte seine Meinung, dass die Erinnerungen von Bredel eine „korrekte, marxistische Interpretation der Ereignisse" lieferten. Unzulässig erschien dem Zensor jedoch die Art, wie der Autor die Beteiligung der Sowjetunion am Krieg in Spanien darstellte. Dies brachte der Gutachter am Schluss seines Gutachtens unmissverständlich, wenn auch in einem recht komplizierten Stil, zum Ausdruck:

Es ist nicht klar genug gesagt, dass die sowjetische Regierung offiziell keine Hilfe leistete, dass sie mit dem sowjetischen Volk übereinstimmte und die individuelle Hilfeleistung nicht verhinderte, aber offiziell keine Hilfe leistete. (AAN GUKPPiW 152-31/121, Gutachten vom 26.1.1950)

Die Veröffentlichung der Erinnerungen von Willi Bredel machte der Gutachter von entsprechenden Eingriffen in den Text auf Seiten 48 und 180-181 abhängig. Aus den überlieferten Akten kann man schließen, dass entsprechende Änderungen im Text der Erinnerungen von Willi Bredel vorgenommen wurden. In einem Gutachten, dass nach dem Erscheinen des Buches gefertigt wurde, stellte der mit der Nachzensur beauftragte Mitarbeiter der Zensurbehörde dem Text eine „sehr positive“ Note aus. Die Erinnerungen von Willi Bredel hätten „ausgezeichnete sozial-erzieherische Werte“, und die Auflage von 10000 Exemplaren sei aus diesem Grunde zu klein. (AAN GUKPPiW 152-31/121, Gutachten vom 26.1.1950)

Zwei andere Titel von Autoren aus der DDR, die den Spanischen Bürgerkrieg behandelten, sorgten bei der Zensur in der Volksrepublik Polen für weit größere Verwirrungen. Der zweiteilige Roman Leutnant Bertram von Bodo Uhse, den der Verlag des Verteidigungsministeriums (MON) gegen Ende des Jahres 1950 dem Hauptamt zur Kontrolle der Presse, Veröffentlichungen und Schaustellungen in Warschau zur Prüfung vorlegte, brachte es auf elf Zensurgutachten, bis er endlich im 
April 1952 zum Druck freigegeben wurde. Weit weniger Gutachten mussten in derselben Zeit im Falle des Romans Grüne Oliven und nackte Berge von Eduard Claudius angefertigt werden, den der parteieigene Verlag Książka i Wiedza (KiW) herausbringen wollte. Dass in diesem Fall das Zensurverfahren bereits nach vier Gutachten abgeschlossen wurde, konnte die Mitarbeiter des Verlages wenig trösten. Das Urteil der Zensurbehörde war eindeutig - der Roman durfte im Jahr 1951 nicht erscheinen.

Der Fall des Romans Leutnant Bertram von Bodo Uhse stellt einen komplizierten, schwierigen, aber auch recht dynamischen und aus heutiger Sicht interessanten Zensurvorgang dar und verdient es, an dieser Stelle etwas ausführlicher behandelt $\mathrm{zu}$ werden. Der Roman konnte trotz einiger negativer Urteile mehrerer Zensoren veröffentlicht werden. Über die eigentlichen Gründe der endgültigen Entscheidung kann man heute nur spekulieren. Die Tatsache, dass Bodo Uhse zu dieser Zeit Vorsitzender des Schriftstellerverbandes der DDR war, hat die Zensoren eher in Verlegenheit gebracht als ihre Arbeit erleichtert, was manche von Ihnen ausdrücklich zur Sprache brachten. Aber selbst diejenigen Zensoren, die es für wichtig hielten, dem polnischen Leserpublikum den Roman eines Deutschen zu präsentieren, der an den Kämpfen in Spanien teilgenommen hatte, stellten dem Buch ein vernichtendes Urteil aus und fanden seine Erscheinung nur unter bestimmten Bedingungen möglich.

Die Intensität, mit der die polnische kommunistische Zensur den Roman von Bodo Uhse prüfte, beweisen insgesamt elf Zensurgutachten. Die einzelnen Zensoren wiesen zwar auf die gleichen Themen- und Problemfelder des Romans hin, aber ihre Beurteilungen gingen oft weit auseinander. Das am meisten von ihnen diskutierte Problem war die Darstellung des faschistischen Militärs und des antifaschistischen (kommunistischen) Lagers vor und während des Spanischen Bürgerkriegs (Band I und II des Romans). Diejenigen Zensoren, die das Buch negativ beurteilten, machten dem Autor dreierlei Vorwürfe. Es irritierte sie vor allem, dass er sowohl bei den Faschisten als auch bei den Kommunisten negative Seiten und Merkmale aufdeckt. Für unzulässig hielten sie, dass er im ,faschistischen Lager“" positive Figuren sieht und die Faschisten nicht deutlich genug anprangert. Es störte sie auch, dass die in dem Roman dargestellten Kommunisten nicht eindeutig positiv oder gar im negativen Lichte erscheinen.

In der ersten Rezension bemerkt der Zensor, dass in dem Roman ,die richtige Beurteilung des besten antifaschistischen Kaders im deutschen Volke" fehlt. Die deutschen Teilnehmer am Spanischen Bürgerkrieg - deutsche Kommunisten, die in den internationalen Brigaden kämpften, seien pessimistisch dargestellt und die „faschistischen Söldner“ nicht in dem Maße entlarvt, wie sie es verdienten (AAN GUKPPiW 151-31/107, Gutachten vom 9.12.1950). Auf politische Fehler in der Handlung des Romans weist auch der zweite Zensor in seinem Gutachten vom 13. Dezember 1950 hin. Einer dieser Fehler bestehe darin, dass der Autor einen „edlen Grafen“ im faschistischen Lager darstellt, der auf wehrlose Menschen nicht schießen will und verrückt wird, als er später die von den faschistischen Einheiten 
verübten Morde sieht. Eine unverdiente Kritik an den deutschen Arbeitern sah der Zensor darin, dass sie im Roman den ,rassistischen Sitten“ huldigen und Judenwitze erzählen (AAN GUKPPiW 151-31/107, Gutachten vom 13.12.1951).

Viele Zensoren warfen dem Autor in diesem Zusammenhang Naturalismus und Psychologismus vor. Die Bemühungen des Schriftstellers, die Wirklichkeit möglichst genau, mit allen Details abzubilden, verursachten ihrer Meinung nach, dass das Bild der Kommunisten sehr „blass“ geworden sei. Ihre „entschieden ideologische Einstellung“ und das Bild des angestrebten Ziels verwischten ihr „rein menschliches Zögern, Schwäche, materielle Schwierigkeiten“ (AAN GUKPPiW 383-31/108, Gutachten vom 17.3.1951). Im gleichen Sinne wird der Roman auch in zwei weiteren Gutachten kritisiert (AAN GUKPPiW 383-31/108, Gutachten vom 16.1.1951, 10.9.1951). Während sich aber nach diesen Gutachten der Naturalismus negativ auf mögliche Interpretationen des Romans auswirken könnte, weil er kein idealisiertes Bild der Kommunisten darstelle, wird er von einem anderen Zensor gerechtfertigt. Der Naturalismus resultiere zwar aus den Bemühungen um eine möglichst getreue Abbildung der Wirklichkeit, betone aber die Grausamkeit des Krieges und die Bestialität der Faschisten (AAN GUKPPiW 383-31/108, Gutachten vom 20.12.1951).

Einige Zensoren signalisierten ein deutliches Ungleichgewicht in der Komposition des ersten Teiles des Romans, das sich darin äußern würde, dass die deutschen Offiziere im Vordergrund und die Kommunisten im Hintergrund stünden. In seiner vernichtenden Rezension vom 10.9.1951 vermisste der Zensor Wörter wie Kommunist, Proletariat, Bourgeoisie, Klassenkampf. Nach der Lektüre des ersten Teiles habe der Leser keine Ahnung „,vom antifaschistischen Kampf des deutschen Proletariats unter der Führung der kommunistischen Partei und vom deutschen Faschismus mit der in seinem Rücken stehenden Bourgeoisie“ (AAN GUKPPiW 383-31/108, Gutachten vom 10.9.1951). Auf dieses Problem machte auch ein anderer Zensor aufmerksam, der drei Monate später sein Gutachten vorlegte. Auch er bemerkte, dass die Tätigkeit und der Kampf der kommunistischen Partei in der Darstellung von Bodo Uhse in einer völligen Isolierung von den deutschen Arbeitern verliefen (AAN GUKPPiW 383-31/108, Gutachten vom 20.12.1951).

Eine andere Meinung über die Darstellung der deutschen Faschisten und Kommunisten im Roman hatten diejenigen Zensoren, die sie positiv beurteilten oder sich für die Veröffentlichung des Romans unter bestimmten Bedingungen aussprachen. Sie verschwiegen, bagatellisierten oder entschuldigten gar die moralischen, ideologischen und politischen Schwächen der Kommunisten. In ihren Gutachten berichteten sie von zielbewussten, disziplinierten deutschen Revolutionären, die trotz einiger Schwächen ein ,zementiertes Kollektiv“ bildeten (AAN GUKPPiW 383-31/113, Gutachten vom 16.04.1951). Gelobt wurde auch der ,gekonnt gezeigte Pathos des Heroismus", der aus dem Internationalismus und dem Verständnis der deutschen Kommunisten resultiere, die um das neue Deutschland kämpfen würden (AAN GUKPPiW 383-31/108, Gutachten vom 12.11.1951). 
Während einige Zensoren die Darstellungsweise der deutschen Offiziere zu „objektiv" fanden, was ihnen eine gute Gelegenheit zur Beanstandung des Romans gab, betonten ihre Kollegen den sittlichen, politischen und ideologischen Verfall der Faschisten. Nach ihrer Meinung lieferte der Autor ein plastisches, an Details reiches, grausames Bild der Faschisten. Die Grausamkeit der militärischen Disziplin, Methoden, die aus den Soldaten gefühllose, nicht denkende, gegen die Vertreter des Fortschritts kämpfende Automaten und Sadisten machten, ihre geistige Zerrissenheit, seien Elemente, aus welchen sich dieses Bild zusammensetze und auf die polnischen Leser einwirke (AAN GUKPPiW 383-31/108, Gutachten vom 10.03.1951).

Die Gemüter der Zensoren spaltete auch das wichtigste Thema des Romans die Teilnahme der deutschen Antifaschisten bzw. Kommunisten am Spanischen Bürgerkrieg. Charakteristisch ist, dass einige Zensoren die Überpräsenz der Deutschen im ganzen Roman zur Sprache brachten. Sie hatten bei der Lektüre des Leutnant Bertram den Eindruck gewonnen, dass die Deutschen überhaupt die einzigen wären, die um die Befreiung Spaniens von der Bedrohung des Faschismus kämpften, und beklagten sich über die "langweiligen Hervorhebungen der Deutschen“ (AAN GUKPPiW 151-31/107, Gutachten vom 13.12.1951). Unter vielen Schilderungen der ,nationalistisch denkenden Deutschen“ (diese Formulierung bezog sich auch auf die Kommunisten) vermissten die Zensoren sehr oft auch das richtige Bild „,des starken spanischen Kämpfers“. Statt die Fahnenflucht der spanischen Kämpfer zu zeigen, sollte der Autor „den Mut des spanischen Volkes“ hervorheben (AAN GUKPPiW 383-31/113, Gutachten vom 16.4.1952). Der Kampf der Spanier - bezeugte ein anderer Zensor entschieden - verliere in der Auffassung von Bodo Uhse den Charakter des Klassenkampfes ( AAN GUKPPiW 383-31/108, Gutachten vom 10.9.1951).

Die Meinungen der Zensoren waren nicht nur in der Erörterung einzelner Fragen unterschiedlich oder sogar widersprüchlich. Auch die allgemeinen, zusammenfassenden Urteile der Zensoren über das Buch fielen weit auseinander und reichten von vernichtender Kritik über zurückhaltende, vorsichtige Noten zu einem beinahe enthusiastischem Lob. Weitgehende Schwierigkeiten mit der eindeutigen Beurteilung des Romans kündigten sich bereits in dem Gutachten vom 16. Januar 1951 an. Der Zensor resümierte sein Urteil mit folgenden Worten:

Wenn man den Standpunkt des Autors berücksichtigt, so scheint es mir, dass das Buch erscheinen könnte, allerdings unter den Bedingungen, dass die Auflage niedrig und der Preis hoch sein werden, und dass es mit einem Vorwort versehen wird, in welchem die Fehler des Buches und der Standpunkt des Schriftstellers erklärt wird. Meinen Vorschlag begründe ich damit, dass der Roman einerseits das faschistische Lager und seine Methoden entlarvt, andererseits aber - trotz des Naturalismus, von dem ich gesprochen habe - viele schöne (Gestalten der) Revolutionäre zeigt. Der Roman eines deutschen Schriftstellers, der sich an dem Bürgerkrieg in Spanien im Rahmen der ErnstThälmann-Brigade beteiligt hat, scheint sehr wichtig für uns zu sein. (AAN GUKPPiW 383-31/108, Gutachten vom 16.01.1951) 
„Der propagandistische und erzieherische Wert des Buches [sind] sehr hoch“, meinte ein anderer Zensor in Bezug auf den ersten Teil des Romans am 7.9.1951 und meldete keine Vorbehalte an (AAN GUKPPiW 383-31/108, Gutachten vom 7.9.1951). Drei Tage später bezeichnete ein weiterer Gutachter beide Teile des Romans als ,ideologisch sehr schwach“ und fügte zum zweiten Teil des Romans hinzu: „an vielen Stellen sogar falsch“ (AAN GUKPPiW 383-31/108, Gutachten vom 10.9.1951). Am 20.12.1951 stellte der Zensor resümierend fest: „ein interessantes Buch, das trotz seiner Fehler einen hohen ideologischen und künstlerischen Wert besitzt" (AAN GUKPPiW 383-31/108, Gutachten vom 20.12.1951). Das letzte dokumentierte Gutachten wurde am 16.4.1952 verfasst. Der Zensor schließt seine Überlegungen mit folgenden Worten ab:

Im Prinzip hat der Autor trotz der oben erwähnten Mängel das Ziel erreicht, er weist auf die politische Bedeutung der Kämpfe in Spanien und die Schwächen des faschistischen Lagers hin, das alle möglichen Methoden aufgreift, weil es die letzten Stunden seiner Existenz ahnt. Auf der anderen Seite zeigt er die Stärke der Arbeiterklasse und des fortschrittlichen Teils der Menschheit im Kampf um soziale Gerechtigkeit und Frieden. Dem deutschen Chauvinismus wird der entschiedene Internationalismus des internationalen Proletariats gegenübergestellt. (AAN GUKPPiW 383-31/113, Gutachten vom 16.4.1952)

Mit diesem Urteil scheint der Fall Leutnant Bertram für die polnische kommunistische Zensur abgeschlossen zu sein. Vieles spricht dafür, dass die endgültige Entscheidung nicht in der Zensurbehörde getroffen wurde, sondern von einer anderen Institution und Instanz (auf einem der Gutachten befindet sich ein Vermerk vom 7.9.1951: „Zulassen zum Druck auf eigene Verantwortung des Obersten Werner“ wahrscheinlich des Chefredakteurs des Verlags).

Die Ereignisse aus der Zeit des Spanischen Bürgerkriegs behandelte auch der vom Parteiverlag KiW im Jahre 1951 zur Kontrolle vorgelegte autobiographische Roman Grüne Oliven und nackte Berge von Eduard Claudius. Der Zensurvorgang hat in diesem Fall einen ähnlichen Verlauf genommen und rief ähnliche Bedenken hervor wie der Roman Leutnant Bertram von Willi Bredel. Der Unterschied zwischen den beiden Fällen besteht offensichtlich darin, dass die Autobiographie von Eduard Claudius in dem Parteiverlag keinen Befürworter hatte, der sich gegen die negativen Urteile der Zensoren durchsetzen und die Verantwortung für den Druck des Buches auf sich nehmen wollte bzw. konnte.

Der Roman Grüne Oliven und nackte Berge wurde innerhalb von wenigen Wochen im Sommer 1951 von vier Zensoren gelesen und auf die Zweckmäßigkeit seiner Erscheinung in Polen überprüft. Bereits der erste Zensor stellte den Antrag auf Nichtzulassung des Romans zum Druck. Er bemängelte unter anderem, dass die Hauptfiguren des Romans, die Kommunisten, sich nicht als solche verhielten stattdessen sei ihr Auftreten von Dekadenz und Pessimismus geprägt. Die „schwermütigen“ Beschreibungen des Krieges stimmten den Leser gegen den Krieg im 
Allgemeinen und nicht gegen den Faschismus im Speziellen. Die vom Zensor beanstandeten „naturalistischen Neigungen“ des Autors würden besonders an jenen Stellen sichtbar, an denen er die Besuche von deutschen Freiwilligen in Bordellen beschreibe oder über ,jüdische Eigenschaften“ spreche (AAN GUKPPiW 376-31/51, Gutachten vom 4.7.1951).

Neun Tage später folgte das zweite Gutachten von einem anderen Zensor, der dem Roman ebenso kritisch gegenüberstand, ihn jedoch nach Durchführung einiger Änderungen im Text zum Druck empfahl. Er stellte fest, dass der Roman gut und „im marxistischen Geiste“ geschrieben sei, auch wenn der Stil des Autors etwas „schwerfällig“ sei. Er meldete zweierlei Bedenken gegen den Text an: Zum einen im Hinblick auf die antisemitischen Äußerungen einer der Hauptfiguren, zum anderen im Hinblick auf die - nach der Meinung des Zensors - unnötigen Informationen über Waffenlieferungen aus der Sowjetunion an Spanien. Die entsprechenden Stellen könne man problemlos aus dem Text entfernen, ohne dass sich dies negativ auf seine literarischen Qualitäten auswirke (AAN GUKPPiW 376-31/51, Gutachten vom 13.7.1951).

Drei Tage nach Fertigstellung des zweiten Gutachtens wurde der dritte Zensor mit der Lektüre des Romans beauftragt. Auf die von seinen Kollegen beanstandeten Aspekte ging er überhaupt nicht ein und äußerte sich sehr positiv sowohl über den Inhalt als auch das literarische Niveau des Romans. Der Zensor bemerkte keine politischen und ideologischen Fehler im Roman und stellte fest, dass der Roman von „hohem sozial-erzieherischem Wert" sei (AAN GUKPPiW 376-31/51, Gutachten vom 4.8.1951). Das letzte Gutachten vom 14. August 1951 stimmte in der Beurteilung des Romans mit dem ersten überein. Die naturalistischen Beschreibungen der Hauptfiguren lassen nach der Meinung des vierten Zensors den Leser eher an „,verkommene Gestalten als an Kommunisten, die sich ihrer Sache bewusst sind" denken. Zum Schluss betonte der Zensor, der Inhalt des Romans sei „erdrückend und sehr pessimistisch", aber versehentlich - oder vielleicht auch vorsichthalber - verzeichnete er seine Entscheidung (genehmigen, nicht genehmigen oder genehmigen nach Änderungen im Text) nicht auf dem Formular, das zur Erstellung des Gutachtens vorgesehen war (AAN GUKPPiW 376-31/51, Gutachten vom 14.8.1951).

In der Zensurbehörde entschied man sich, den Roman nicht erscheinen zu lassen. Die Entscheidung wurde mit dem Vertreter des Verlages besprochen - es sollte ein Exemplar mit Vorwort herausgebracht werden (AAN GUKPPiW 376-31/51, Vermerk vom 11.9.1951).

Den zweiten Versuch, den Roman zu veröffentlichen, unternahm der Verlag sieben Jahre später, mehr als ein Jahr nach den Ereignissen des Polnischen Oktobers von 1956. Von diesem Zensurvorgang konnte bei Archivrecherchen nur ein Gutachten ermittelt werden. Es ließ sich nicht eindeutig feststellen, ob das eingereichte Manuskript eine gemäß den Vorschlägen des Zensors aus dem Jahre 1951 durch entsprechende Streichungen im Text veränderte Fassung war, oder ob es wieder in 
seiner Originalfassung vorgelegt wurde. Von Pessimismus und Naturalismus, die dem Roman nach Ansicht der Zensoren von 1951 eigen seien, ist im Gutachten vom 26. Februar 1958 nicht mehr die Rede. Stattdessen vergleicht der Zensor Claudius' Roman mit dem Arc de Triomphe von Erich Maria Remarque. Der Autor habe dem hoffnungslosen Dasein von Rawik das Leben des „erbitterten Kämpfers für Freiheit und soziale Gerechtigkeit" gegenübergestellt. Leider sei die künstlerische Form des Romans, die großteils Remarque nachahme, dieser Aufgabe nicht gewachsen. Trotz dieser kritischen Bemerkung stellte der Zensor den Antrag auf die Zulassung des Romans zum Druck (AAN GUKPPiW 598-68/5, Gutachten vom 26.2.1958).

Die Zulassung des Romans ist als ein deutliches Zeichen der Zeit zu sehen. Ein literarisches Werk, in diesem Fall das eines kommunistischen Autors, das bis 1956 aus kulturpolitischen und ideologischen Gründen die Schranken der Zensur nicht passieren konnte, schaffte es 1958 ohne Schwierigkeiten.

\section{Bibliographie}

\section{Archivalien: Archiwum Akt Nowych; Bestand: Główny Urząd Kontroli Prasy Publikacji i Widowisk (AAN GUKPPiW)}

AAN GUKPPiW 375 (31/27). (2. Juli 1951). [Gutachten zu Anna Seghers, Siódmy krzyż (Das siebte Kreuz), Warszawa: Czytelnik 1951], o.S

AAN GUKPPiW 152 (31/120). (2. Januar 1951). [Gutachten zu Willi Bredel, Spotkanie nad Ebro (Begegnung am Ebro. Aufzeichnungen eines Kriegkommissars), Warszawa: PIW 1949/50], 93-94.

AAN GUKPPiW 376 (31/51). (4. Februar 1951). [Gutachten zu Eduard Claudius, Zielone oliwki i nagie skaty (Grüne Oliven und nackte Berge), Warszawa: KiW 1951], 449-450.

AAN GUKPPiW 375 (31/27). (2. Juli 1951). [Gutachten zu Anna Seghers, Siódmy krzyż (Das siebte Kreuz), Warszawa: Czytelnik 1951], o.S.

AAN GUKPPiW 152 (31/120). (2. Januar 1950). [Gutachten zu Willi Brede, Spotkanie nad Ebro (Begegnung am Ebro. Aufzeichnungen eines Kriegkommissars), Warszawa: PIW 1949/50], 93-94.

AAN GUKPPiW 152 (31/121). (26. Januar 1950). [Gutachten zu Willi Bredel, Spotkanie nad Ebro (Begegnung am Ebro. Aufzeichnungen eines Kriegkommissars), Warszawa: PIW 1949/50], 75-77.

AANGUKPPiW 151 (31/107). (9. Dezember 1950). [Gutachten zu Bodo Uhse, Leutnant Bertram (Leutnant Bertram), Warszawa MON 1951], 239.

AANGUKPPiW 151 (31/107). (13. Dezember 1950). [Gutachten zu Bodo Uhse, Leutnant Bertram (Leutnant Bertram), Warszawa MON 1951], 240-243.

AANGUKPPiW 151 (31/108). (16. Januar 1951). [Gutachten zu Bodo Uhse, Leutnant Betram (Leutnant Bertram), Warszawa MON 1951], 57.

AANGUKPPiW 151 (31/108). (10. März 1951). [Gutachten zu Bodo Uhse, Leutnant Betram (Leutnant Bertram), Warszawa MON 1951], 58.

AANGUKPPiW 151 (31/108). (17. März 1951). [Gutachten zu Bodo Uhse, Leutnant Betram (Leutnant Bertram), Warszawa MON 1951], 58a.

AANGUKPPiW 151 (31/108). (10. September 1951). [Gutachten zu Bodo Uhse, Leutnant Betram (Leutnant Bertram), Warszawa MON 1951], 59.

AANGUKPPiW 151 (31/108). (12. November 1951). [Gutachten zu Bodo Uhse, Leutnant Betram (Leutnant Bertram), Warszawa MON 1951], 60-61. 
AANGUKPPiW 151 (31/108). (8. Dezember 1951). [Gutachten zu Bodo Uhse, Leutnant Betram (Leutnant Bertram), Warszawa MON 1951], 63.

AANGUKPPiW 151 (31/108). (20. Dezember 1951). [Gutachten zu Bodo Uhse, Leutnant Betram (Leutnant Bertram), Warszawa MON 1951], 62.

AANGUKPPiW 151 (31/113). (16. April 1952). [Gutachten zu Bodo Uhse, Leutnant Betram (Leutnant Bertram), Warszawa MON 1951], 27.

AAN GUKPPiW 376 (31/51). (4. Juli 1951). [Gutachten zu Eduard Claudius, Zielone oliwki i nagie skaty (Grüne Oliven und nackte Berge), Warszawa KiW 1951], 449-450.

AAN GUKPPiW 376 (31/51). (13. Juli 1951). [Gutachten zu Eduard Claudius, Zielone oliwki i nagie skaty (Grüne Oliven und nackte Berge), Warszawa KiW 1951], 451-452.

AAN GUKPPiW 376 (31/51). (4. August 1951). [Gutachten zu Eduard Claudius, Zielone oliwki i nagie skaty (Grüne Oliven und nackte Berge), Warszawa KiW 1951], 453-454.

AAN GUKPPiW 376 (31/51). (14. August 1951). [Gutachten zu Eduard Claudius, Zielone oliwki i nagie skaty (Grüne Oliven und nackte Berge), Warszawa KiW 1951], 455-456.

AAN GUKPPiW 376 (31/51). (4. September 1951). [Gutachten zu Eduard Claudius, Zielone oliwki i nagie skaty (Grüne Oliven und nackte Berge), Warszawa KiW 1951], 449-456.

AAN GUKPPiW 598 (68/5). (26. Februar 1958). [Gutachten zu Eduard Claudius, Zielone oliwki i nagie skaty (Grüne Oliven und nackte Berge), Warszawa KiW 1958], 16.

\section{Sonstige Literatur}

Rajch, M. (2015). Unsere andersartige Kulturpolitik: Zensur und Literatur in der DDR und in der Volksrepublik Polen. Poznań: Wydawnictwo Naukowe UAM. 